\title{
Stress-Strain Relationships in Yarns Subjected to Rapid Impact Loading: 2. Breaking Velocities, Strain Ener- gies, and Theory Neglecting Wave Propagation ${ }^{1}$
}

\author{
Frank L. McCrackin, Herbert F. Schiefer, Jack C. Smith, and Walter K. Stone
}

\begin{abstract}
The behavior of a yarn specimen fastened at one end to a head mass and at the other end to a small tail mass is analyzed for longitudinal impact of the specimen at the head. The analysis leads to a basic formula for "limiting breaking velocity," which is a characteristic property of the material and independent of the dimensions of the specimen. A simple procedure is described for obtaining its value. The values for cotton, nylon, and undrawn nylon yarns tested at room temperature are found to be 130, 228, and 550 meters per second, respectively. The practical application of the limiting breaking velocity to safety engineering is shown by an example on safety lines. Formulas are derived for computing energy to any strain under impact loading, and the computed values are found to agree with those obtained from the area under the impact load-extension curves.
\end{abstract}

\section{Introduction}

The first paper of this series [1] ${ }^{2}$ describes equipment and procedures for studying the stress-strain behavior of a yarn specimen subjected to tensional impact. In a typical test, a $65-\mathrm{cm}$ specimen was attached at one end to a head mass and at the other end to a tail mass. The head was impacted at velocities between 10 and $100 \mathrm{~m} / \mathrm{sec}$, and subsequent behavior was recorded by high-speed photography.

The behavior of the yarn specimen after longitudinal impact is treated theoretically in this paper, neglecting wave-propagation effects. The results are compared with those obtained experimentally for several materials. The practical application of this work to safety engineering is indicated.

\section{Theory}

Figure 1 represents a test specimen terminated by a head and a tail. The equations of motion of these masses are derived in terms of the following symbols:

\section{$L=$ Length of specimen before impact.}

$w=$ Mass of specimen.

$n w=$ Mass of tail.

$m w=$ Mass of head

$x_{10}=$ Position of tail before impact, relative to a fixed origin, 0 .

$x_{20}=$ Position of head before impact, relative to a fixed origin, $\mathrm{O}$.

$t=$ Time after impact

$x_{1}=$ Position of tail at time, $t$, relative to a fixed origin, $\mathrm{O}$.

$x_{2}=$ Position of head at time, $t$, relative to a fixed origin, $\mathrm{O}$.

$x_{c}=$ Position of center of mass of the system at time, $t$, relative to a fixed origin, $\mathrm{O}$.

$\epsilon=$ Strain in specimen.

$F=$ Force acting on specimen at time, $t$.

$v_{0}=$ Velocity of head immediately after impact.

It is assumed here that $\frac{1}{2} w$ is concentrated at the head and the remaining $\frac{1}{2} w$ is concentrated at the tail of the specimen. Thus the problem ac-

1 This work was sponsored by the Office of the Quartermaster General, Department of the Army, and was presented in part at the September 1953 and 1954 meetings of The Fiber Society, and also at the November 1954 meeting of the Society of Rheology.

2 Figures in brackets indicate the literature references at the end of this paper.

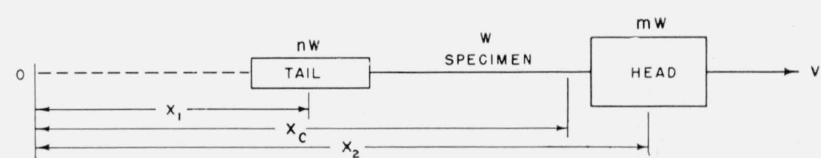

FIGURE 1. Schematic drawing of test specimen for impact loading.

tually treated is that of a mass-less specimen to which a head mass $\left(m+\frac{1}{2}\right) w$ and a tail mass $\left(n+\frac{1}{2}\right) w$ are attached.

The force equations, neglecting air friction, ${ }^{3}$ are

$$
F=\left(n+\frac{1}{2}\right) w \frac{d^{2} x_{1}}{d t^{2}},
$$

and

$$
F=-\left(m+\frac{1}{2}\right) w \frac{d^{2} x_{2}}{d t^{2}}
$$

From definition of the center of mass, we obtain $\left(n+\frac{1}{2}\right) w x_{1}+\left(m+\frac{1}{2}\right) w x_{2}=(n+m+1) w x_{c}$.

Differentiating and eliminating $w$ gives

$$
\left(n+\frac{1}{2}\right) \frac{d x_{1}}{d t}+\left(m+\frac{1}{2}\right) \frac{d x_{2}}{d t}=(n+m+1) \frac{d x_{c}}{d t} .
$$

Because $\epsilon L=\left(x_{2}-x_{1}\right)-\left(x_{20}-x_{10}\right)$, we obtain by differentiation

$$
\frac{d x_{1}}{d t}-\frac{d x_{2}}{d t}=-L \frac{d \epsilon}{d t}
$$

Solving eq (3) and (4) for $d x_{1} / d t$ and $d x_{2} / d t$ gives

$$
\frac{d x_{1}}{d t}=\frac{d x_{c}}{d t}-\frac{\left(m+\frac{1}{2}\right) L}{n+m+1} \frac{d \epsilon}{d t}
$$

and

$$
\frac{d x_{2}}{d t}=\frac{d x_{c}}{d t}+\frac{\left(n+\frac{1}{2}\right) L}{n+m+1} \frac{d \epsilon}{d t}
$$

3 The force on the tail due to air friction was calculated to be approximately 1 percent of the yarn tension. 
At maximum strain $\epsilon=\epsilon_{m}$ and $d \epsilon / d t=0$, therefore, $d x_{1} / d t=d x_{2} / d t=d x_{c} / d t$. That is, for a typical loading and unloading test under impact conditions, the velocity of the tail mass at the instant of maximum strain is equal to the velocity of the head, and both are equal to the velocity of the center of the total mass. As the total momentum imparted by the hammer to the head immediately after impact is $\left(m+\frac{1}{2}\right) w v_{0}$, it follows from conservation of momentum that $d x_{c} / d t=\left(m+\frac{1}{2}\right) v_{0} /(n+m+1)=$ constant, which is also the velocity of the head and of the tail at maximum strain. The velocity of the head, therefore, decreases from $v_{0}$ immediately after impact to $\left(m+\frac{1}{2}\right) v_{0} /(n+m+1)$ at the time of maximum strain. It is seen that this decrease is equal to $\left(n+\frac{1}{2}\right) v_{0} /(n+m+1)$ and thus depends upon the values of $n$ and $m$.

From eq (5) and (1), or (6) and (2), it follows that

$$
\frac{d^{2} \epsilon}{d t^{2}}=-\frac{n+m+1}{\left(n+\frac{1}{2}\right)\left(m+\frac{1}{2}\right)} \frac{F}{L w} .
$$

Integration gives

$$
\left(\frac{d \epsilon}{d t}\right)^{2}=-\frac{2}{L w} \frac{n+m+1}{\left(n+\frac{1}{2}\right)\left(m+\frac{1}{2}\right)} \int_{0}^{\epsilon} F d \epsilon+C .
$$

At $t=0, \epsilon=0$, and $d \epsilon / d t=v_{0} / L$. Therefore, $\mathrm{C}=$ $v_{0}^{2} / L^{2}$ and

$$
\left(\frac{d \epsilon}{d t}\right)^{2}=v_{0}^{2}-\frac{2}{L w} \frac{n+m+1}{\left(n+\frac{1}{2}\right)\left(m+\frac{1}{2}\right)} \int_{0}^{\epsilon} F d \epsilon .
$$

At the maximum strain $\epsilon=\epsilon_{m}$ and $d \epsilon / d t=0$. follows, therefore, that

$\log v_{0}=-\frac{1}{2} \log \frac{\left(n+\frac{1}{2}\right)\left(m+\frac{1}{2}\right)}{n+m+1}+\log \sqrt{\frac{2 L}{w} \int_{0}^{\epsilon_{m}} F d \epsilon .}$

In an impact test the specimen will break if the impact velocity, $v_{0}$, is large enough that the rupture strain, $\boldsymbol{\epsilon}_{\tau}$, is attained. The minimum breaking velocity, $v_{n}$, for a given $n$ is the velocity just sufficient to cause the specimen to break when $\boldsymbol{\epsilon}_{r}=\boldsymbol{\epsilon}_{m}$. Under these conditions we have

$\log v_{n}=-\frac{1}{2} \log \frac{\left(n+\frac{1}{2}\right)\left(m+\frac{1}{2}\right)}{n+m+1}+\log \sqrt{\frac{2 L}{w} \int_{0}^{\epsilon_{r}} F d \epsilon .}$

Equation (12) expresses the minimum breaking velocity as a function of $n$. If $\epsilon_{r}$ and the shape of the stress-strain curve are both independent of the rate of straining, the expression under the radical sign is a constant having the dimensions of velocity squared. At high rates of straining the value of $\epsilon_{r}$ is less than that obtained at conventional testing rates. Also, the slope of the stress-strain curve is greater at higher rates of straining. However, for rates of straining: of the same order of magnitude, these changes are small, and their effects compensate each other. Consequently, the area under the stress-strain curve should be essentially constant for the rates of straining considered here. Under these conditions a plot of $\log v_{n}$ versus $\log \left(n+\frac{1}{2}\right)\left(m+\frac{1}{2}\right) /(n+m+1)$ would be a straight line of slope $-\frac{1}{2}$.

By extrapolating from impact test data to the point at which $\log \left(n+\frac{1}{2}\right)\left(m+\frac{1}{2}\right) /(n+m+1)=0$, a characteristic velocity, $v_{b}$, is obtained; namely,

$$
v_{b}=\sqrt{\frac{2 L}{w} \int_{0}^{\epsilon_{r}} F d \epsilon}
$$

If we let $\rho=$ density of the specimen and $\sigma=$ stress, eq (13) reduces to the form

$$
v_{b}=\sqrt{\frac{2}{\rho} \int_{0}^{\epsilon_{r}} \sigma d \epsilon .}
$$

It is thus seen that $v_{b}$ is a quantity characteristic of the material itself, except for a possible dependence on the rate of straining.

Equation (14) can be rearranged in the form

$$
\frac{1}{2} \rho v_{\mathrm{b}}^{2}=\int_{0}^{\epsilon_{r}} \sigma d \epsilon,
$$

which states that the kinetic-energy density in the specimen when traveling at velocity $v_{b}$ is just equal to the strain-energy density required to break the specimen. We thus see that if the specimen is impacted at a velocity greater then $v_{b}$, it will always be broken. For this reason, we call $v_{b}$ the limiting breaking velocity.

Von Kármán [2] derived a critical velocity, $v_{c}$, at which a filament will break immediately upon impact

$$
v_{c}=\int_{0}^{\epsilon_{r}} \sqrt{\frac{1}{\rho} \frac{d \sigma}{d \epsilon}} d \epsilon
$$

This equation was obtained from a consideration of plastic and elastic wave propagation in a material for which the stress-strain curve is concave downward, and the stress is independent of the rate of straining.

The results calculated from eq (14) and (16) differ slightly. However, in the special case when Hooke's law is obeyed both eq (14) and (16) reduce to

$$
v^{\prime}=\epsilon_{r} \sqrt{\frac{E}{\rho}} .
$$

This formula may be expressed in terms of the more familiar textile quantities of tenacity and percent elongation at rupture, namely,

$$
v^{\prime}=29.7 \sqrt{\text { tenacity } \times \text { elongation },}
$$

where $v^{\prime}$ is in meters per second, tenacity is in grams per denier, and elongation is in percent.

Meredith [3] has obtained evidence that at high rates of straining the load-extension curves of some materials become more Hookean. These formulas thus assume considerable importance and by means of eq (18), estimates of the critical velocities of yarns may be computed from their tenacities and elongations at rupture under impact testing conditions. 


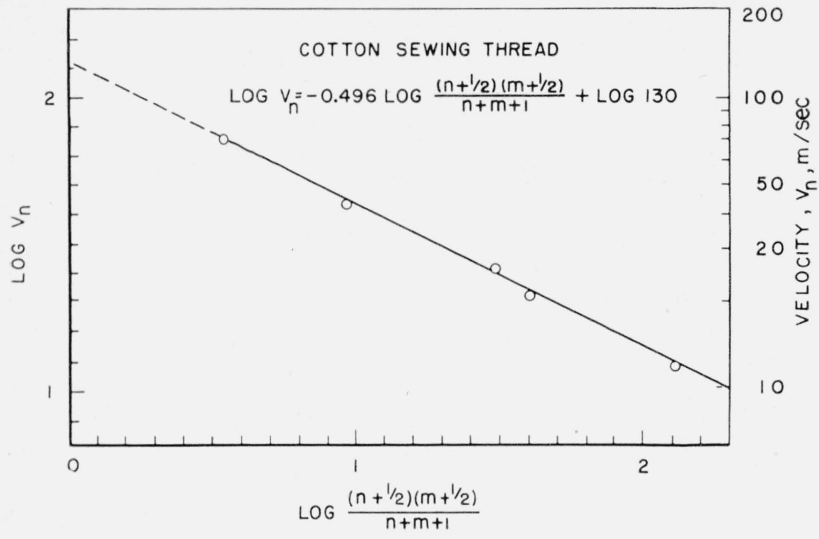

FIGURE 2. Relationship between impact velocity to rupture, $v_{n}$, and head-tail mass parameter, $\left(n+\frac{1}{2}\right)\left(m+\frac{1}{2}\right) /(n+m+1)$, for cotton sewing thread.

In a subsequent paper [4] the behavior of the specimen after longitudinal impact is treated by a theory that considers the effects of wave propagation. This treatment shows that the results given in this paper for nonwave theory are valid when the tail mass is greater than $40 w$. For tail masses less than $40 w$, the effects of wave propagation may become appreciable and must be considered in the theoretical treatment.

\section{Experimental Results}

Experimental values of $v_{n}$ corresponding to different values of $n$ were obtained on a number of different materials. These values of $\log v_{n}$ are plotted in figures 2, 3, and 4 against $\log \left(n+\frac{1}{2}\right)\left(m+\frac{1}{2}\right) /$ $(n+m+1)$. These plots are straight lines having the empirical equations

$$
\log v_{n}=-0.496 \log \frac{\left(n+\frac{1}{2}\right)\left(m+\frac{1}{2}\right)}{n+m+1}+\log 130
$$

for cotton thread

$$
\log v_{n}=-0.495 \log \frac{\left(n+\frac{1}{2}\right)\left(m+\frac{1}{2}\right)}{n+m+1}+\log 228
$$

for nylon yarn and threads

$$
\log v_{n}=-0.500 \log \frac{\left(n+\frac{1}{2}\right)\left(m+\frac{1}{2}\right)}{n+m+1}+\log 550
$$

for undrawn nylon.

It should be noted that the slope of each line represented by these equations is very close to $-\frac{1}{2}$ in accordance with eq (12) for $\log v_{n}$.

\section{Design of Safety Lines}

The empirical equation derived for $\log v_{n}$ has many practical applications. Consider, for example, a safety line of length, $L$, that connects a construction worker to a rigid point of the structure. Under these conditions, $m$ is infinite. The maximum free

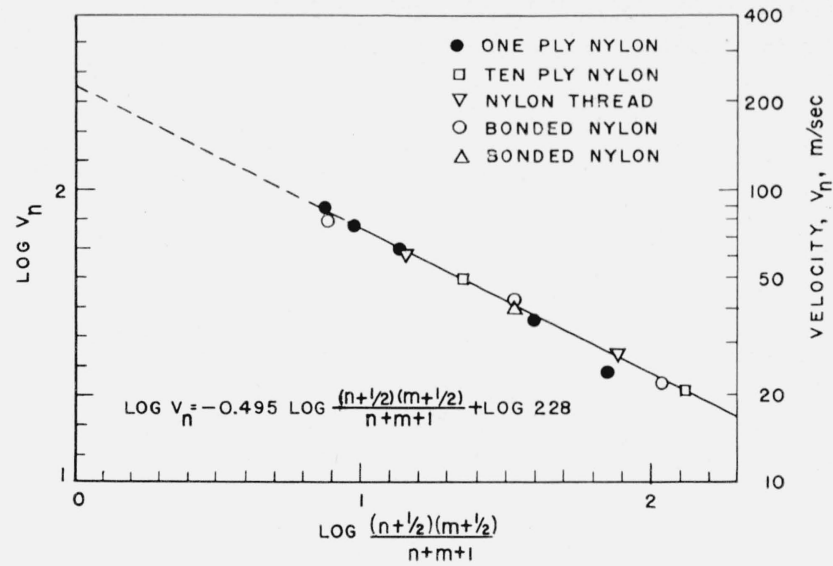

FIGURE 3. Relationship between impact velocity to rupture, $v_{n}$, and head-tail mass parameter, $\left(n+\frac{1}{2}\right)\left(m+\frac{1}{2}\right) /(n+m+1)$, for nylon yarns and threads.

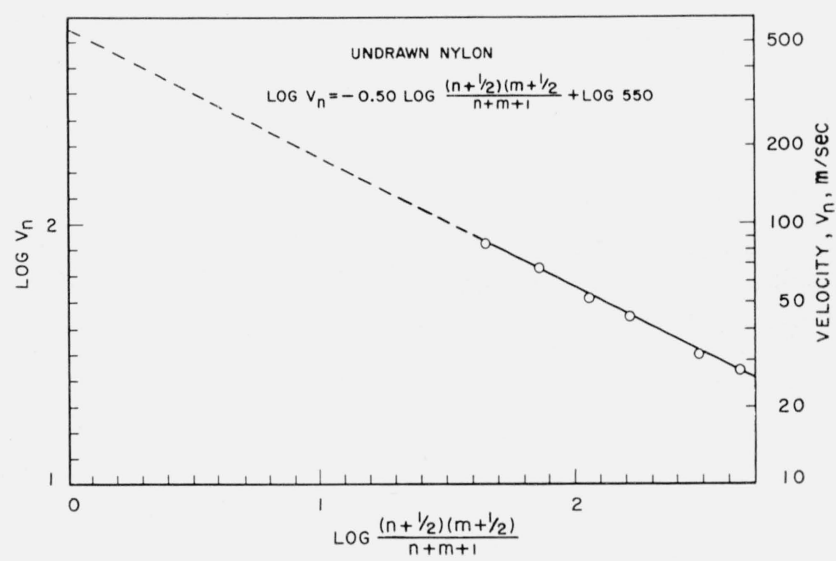

FICURE 4. Relationship between impact velocity to rupture, $v_{n}$, and head-tail mass parameter, $\left(n+\frac{1}{2}\right)\left(m+\frac{1}{2}\right) /(n+m+1)$, for undrawn nylon.

fall of the worker would be $2 L$, and the maximum free-fall velocity $2 \sqrt{g L}$. As $m$ becomes infinite, eq (12) reduces to

$$
\log v_{n}=-\frac{1}{2} \log \left(n+\frac{1}{2}\right)+\log v_{b} .
$$

Making the assumption (which must be checked by experiment) that the safety line behaves under impact like the single yarn considered in the derivation of eq (19), substituting $v_{n}=2 \sqrt{g L}$ and solving for $n$, one obtains

$$
n=\frac{v_{b}^{2}-2 g L}{4 g L} .
$$

If $v_{b}$ is $200 \mathrm{~m} / \mathrm{sec}$ for the material of the safety line, $L=10 \mathrm{~m}$, and $g=9.8 \mathrm{~m} / \mathrm{sec}^{2}$, the computed value of $n$ is 101 . If the weight of the worker, $n w$, is $202 \mathrm{lb}$, the minimum weight of $10 \mathrm{~m}$ of safety line, $w$, to just support the worker at impact is $2 \mathrm{lb}$, according to this computation.

This weight is approximately that of a rope $1 / 2 \mathrm{in}$. in diameter [5]. A nylon rope $7 / 16$ in. in diameter has a static breaking strength of $4,000 \mathrm{lb}$ [6], or 20 times 
the weight of the worker. However, under the dynamic conditions just assumed, a nylon rope with $v_{b}=200 \mathrm{~m} / \mathrm{sec}$ would have no factor of safety whatever according to this computation.

If $v_{b}$ for the material of the safety line is 100 , $n=25$, and for the same weight of worker, $w$ would have to be $8 \mathrm{lb}$. This weight is approximately that of a rope about $1 \mathrm{in}$. in diameter [5]. A manila rope 1 in. in diameter has a static breaking strength of $11,000 \mathrm{lb}$, or 50 times the estimated weight of the worker. Again, under dynamic conditions, this manila rope would not provide a reasonable factor of safety according to this computation. Results obtained by Newman [6] showed that a $9 / 16$-in.-diam sisal rope $10 \mathrm{ft}$ long broke when a weight of $142 \mathrm{lb}$ was dropped a height of only $20 \mathrm{ft}$.

\section{Energy of Straining}

The energy per unit length, $Q$, to stress the specimen to a strain $\epsilon$ is given from eq (10) by the equation

$$
Q=\int_{0}^{\epsilon} F d \epsilon=\frac{L w}{2} \frac{\left(n+\frac{1}{2}\right)\left(m+\frac{1}{2}\right)}{n+m+1}\left[\left(\frac{v_{0}}{L}\right)^{2}-\left(\frac{d \epsilon}{d t}\right)^{2}\right],
$$

where $d \epsilon / d t$ is the slope of the strain-time curve at the strain $\epsilon$. If the specimen is not ruptured but is loaded to a maximum strain, $\epsilon_{m}$, and then unloaded, the energy, $Q_{m}$, to the maximum strain, $\boldsymbol{\epsilon}_{m}$, is given by the equation

$$
Q_{m}=\int_{0}^{\epsilon_{m}} F d \epsilon=\frac{L w}{2} \frac{\left(n+\frac{1}{2}\right)\left(m+\frac{1}{2}\right)}{n+m+1}\left(\frac{v_{0}}{L}\right)^{2},
$$

since $(d \epsilon / d t)_{\epsilon=\epsilon_{m}}$ is zero. If the specimen is ruptured, the energy, $Q_{r}$, to the rupture strain, $\epsilon_{r}$, is given by the equation

$Q_{r}=\int_{0}^{\epsilon_{r}} F d \epsilon=\frac{L w}{2} \frac{\left(n+\frac{1}{2}\right)\left(m+\frac{1}{2}\right)}{n+m+1}\left[\left(\frac{v_{0}}{L}\right)^{2}-\left(\frac{d \epsilon}{d t}\right)_{\epsilon=\epsilon_{r}}^{2}\right]$

The value of $(d \epsilon / d t)_{\epsilon=\epsilon_{r}}$ will be zero, if the impact velocity of the head is just sufficient to produce rupture. Above this velocity $(d \epsilon / d t)_{\epsilon=\epsilon_{r}}$ is a positive quantity.

By means of eq (20), (21), and (22), it is possible to compute $Q, Q_{m}$, and $Q_{r}$ from the conditions of a test. For $Q_{m}$ it is only necessary to know the velocity, $v_{0}$, of the head at impact. For $Q$ and $Q_{r}$, the value of $d \epsilon / d t$ and $(d \epsilon / d t)_{\epsilon=\epsilon_{r}}$ must be known in addition to the value of $v_{0}$. The values of $v_{0}, d \epsilon / d t$, and $(d \epsilon / d t)_{\epsilon=\epsilon_{r}}$ are readily obtained from the positions of the head and of the tail on the high-speed motion pictures taken during the test. The energy values computed by means of these equations for different materials and impact testing conditions are plotted in figure 5 against those derived from the area of the impact-load-extension curves. The plotted points fall near a straight line having a slope equal to 1.

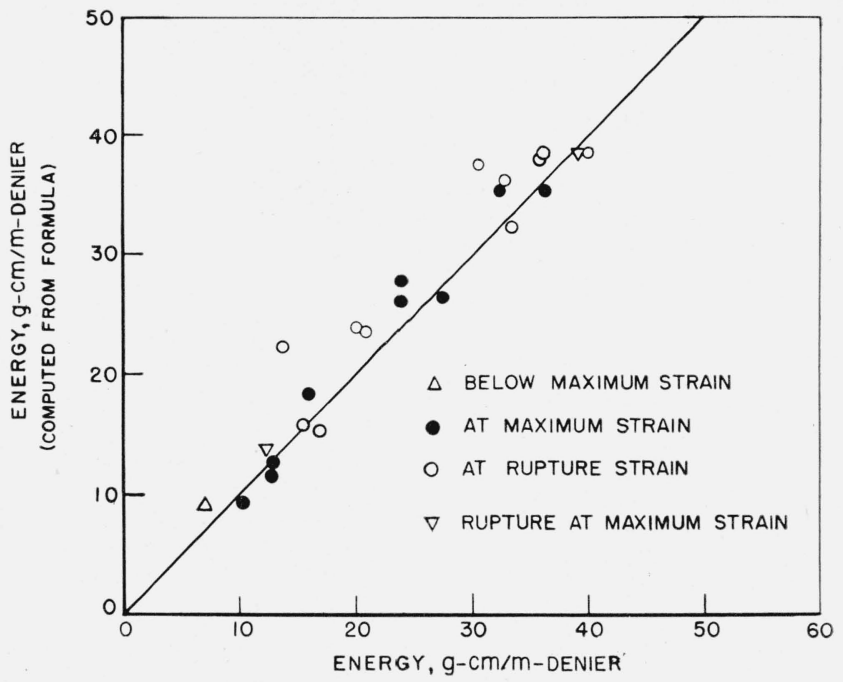

(COMPUTED FROM AREA OF LOAD-ELONGATION CURVE)

FiguRE 5. Energy values computed from formulas plotted against those based upon the area under the impact loadextension curves.

\section{Summary and Conclusions}

The theoretical treatment given in this paper for longitudinal impact of a yarn leads to a formula for the limiting breaking velocity that is a characteristic property of the material and is independent of the dimensions of the specimen. A specimen impacted at a velocity greater than its limiting breaking velocity will always be broken. A simple procedure is described for obtaining this limiting breaking velocity from impact-test data, and results are given for cotton, nylon, and undrawn nylon yarns. The potential importance of the limiting breaking velocity to safety engineering is indicated by its application to estimates of margin of safety of rupture of safety lines for construction workers. The theoretical treatment also leads to formulas for obtaining energy to any given strain, including rupture strain, under different impact conditions. These values agree reasonably well with those obtained from the area under the impact-load-extension curves.

\section{References}

[1] Walter K. Stone, Herbert F. Schiefer, and George Fox, Stress-strain relationships in yarns subjected to rapid impact loading: 1. Equipment, testing procedure, and typical results, J. Research NBS 54, 269 (1955) RP2589.

[2] T. von Kármán and P. Duwez, Propagation of plastic deformation in solids, J. Appl. Phys. 21, 987 (1950).

[3] R. Meredith, The effect of rate of extension on the tensile behavior of viscose and acetate rayons, silk, and nylon, J. Textile Inst. 45, T30 (1954).

[4] Jack C. Smith, Frank L. McCrackin, and Herbert F. Schiefer, Stress-strain relationships in yarns subjected to rapid impact loading: 3 . Effect of wave propagation, J. Research NBS $\mathbf{5 5}$ (in press).

[5] Sanford B. Newman and John H. Curtiss, A statistical analysis of some mechanical properties of manila rope, J. Research NBS 39, 551 (1947) RP1847.

[6] Sanford B. Newman and Helen G. Wheeler, Impact strength of nylon and sisal ropes, J. Research NBS 35, 417 (1945) RP1679.

Washington, December 17, 1954. 